\title{
8. Altern nachfolgende Kohorten gesünder? Selbstberichtete Erkrankungen und funktionale Gesundheit im Kohortenvergleich
}

\author{
Julia K. Wolff, Sonja Nowossadeck \& Svenja M. Spuling
}

\section{Kernaussagen}

Trotz eines hohen Anteils von Personen mit Mehrfacherkrankungen berichten viele der 40- bis 85-Jährigen im Jahr 2014 von einer guten funktionalen Gesundheit: Bereits die 40- bis 54-Jährigen sind 2014 etwa zur Hälfte (47,1 Prozent) von mehr als zwei Erkrankungen betroffen. Bei den 55- bis 69-Jährigen und 70- bis 85-Jährigen sind es mit 65,5 und 82,1 Prozent deutlich mehr. Gleichzeitig berichtet die Mehrheit der 40- bis 85-Jährigen von einer guten funktionalen Gesundheit (68,9 Prozent). Selbst Personen mit fünf und mehr Erkrankungen geben zu 39,2 Prozent noch eine gute funktionale Gesundheit an.

Bei der Gesundheit bestehen im Jahr 2014 große Bildungsunterschiede: Niedriggebildete berichten im Jahr 2014 seltener von höchstens einer Erkrankung (23,0 Prozent) sowie seltener von einer guten funktionalen Gesundheit (45,8 Prozent) als Personen mit mittlerer Bildung (37,1 Prozent höchstens eine Erkrankung; 65,4 Prozent gute funktionale Gesundheit) oder Hochgebildete (41,6 Prozent höchstens eine Erkrankung; 79,1 Prozent gute funktionale Gesundheit).

Der Wandel hin zu weniger selbstberichteten Erkrankungen von 1996 zu 2008 setzt sich im Jahr 2014 nicht fort: Von 1996 bis 2002 nannte ein größer werdender Anteil der 40- bis 85-Jährigen keine oder nur eine Erkrankung. Während der Anteil von Personen mit höchstens einer Erkrankung von 2002 bis 2008 bei circa 44 Prozent stabil blieb, ist er zwischen 2008 und 2014 um sechs Prozentpunkte gesunken - liegt allerdings noch zwei Prozentpunkte über dem Niveau von 1996. Dieser Wandel betrifft alle Altersgruppen gleichermaßen.

Der Anteil an Personen mit guter funktionaler Gesundheit hat zwischen 2008 und 2014 zugenommen, allerdings nur bei den über 65-Jährigen: Personen im Alter von 66 bis 83 Jahren berichten 2014 häufiger von einer guten funktionalen Gesundheit als noch im Jahr 2008. Dagegen hat der Anteil an Personen mit guter funktionaler Gesundheit unter den unter 66-Jährigen zwischen 2008 und 2014 abgenommen.

\subsection{Einleitung}

Mit zunehmendem Alter spielt die Aufrechterhaltung einer guten Gesundheit nicht nur für das Wohlbefinden oder für persönliche Aktivitäten (vgl. Kapitel 11 und 17) eine bedeutende Rolle, sondern auch für die gesellschaftliche
Teilhabe, wie das ehrenamtliche Engagement oder die Erwerbstätigkeit (vgl. Kapitel 3 und 5). Gute Gesundheit umfasst dabei nicht nur die Abwesenheit von Krankheit sondern mehrere Dimensionen. Die Weltgesundheitsorganisation 
(WHO) definiert Gesundheit zum Beispiel „als Zustand des vollkommenen körperlichen, seelischen und sozialen Wohlbefindens und nicht als die bloße Abwesenheit von Krankheit oder Gebrechen“ (WHO 1946: 1). Häufig werden in der Erforschung der Gesundheit vier Facetten unterschieden: subjektive, psychische, körperliche und funktionale Gesundheit.

Die subjektive Gesundheit ist die individuelle Bewertung des Gesundheitszustands einer Person und ein bedeutender Prädiktor für Lebensqualität. Sie wird normalerweise mit einer einzelnen Frage „Wie schätzen Sie Ihren derzeitigen Gesundheitszustand ein?" von ,sehr schlecht" bis ,sehr gut ' erfasst. Die subjektive Gesundheit wird in Kapitel 10 genauer thematisiert. Die psychische Gesundheit umfasst verschiedene psychische Erkrankungen wie zum Beispiel Depressionen. Depressive Symptome als Ausdruck der negativen Befindlichkeit werden in Kapitel 11 untersucht. Das vorliegende Kapitel beschäftigt sich dagegen näher mit der körperlichen und funktionalen Gesundheit und verfolgt dabei zwei zentrale Fragen: Wie ist die Gesundheit im Jahr 2014 und wie hat sich die Gesundheit seit 1996 gewandelt?

Die körperliche Gesundheit wird durch die Erkrankungen einer Person beschrieben und auch unter dem Begriff der Morbidität gefasst. Je nach Definition gilt die Kombination mehrerer (mindestens zwei) chronischer Erkrankungen als Multimorbidität. Häufig, wie auch im Deutschen Alterssurvey (DEAS), werden dazu selbstberichtete Erkrankungen herangezogen, die ein ähnlich gutes Maß für die körperliche Gesundheit sind wie von Ärzten diagnostizierte Erkrankungen (Katz, Chang, Sangha, Fossel, \& Bates 1996). Zudem wird im DEAS als ein medizinisch objektiver Indikator die Lungenfunktion als zusätzliches Maß der körperlichen Gesundheit und Fitness erhoben.

Die funktionale Gesundheit bezieht sich auf Einschränkungen in der Mobilität oder den Alltagskompetenzen. Diese funktionalen Einschränkungen sind neben der Anzahl der körperlichen Erkrankungen von zentraler Bedeutung. Denn körperlich krank zu sein heißt nicht unbedingt auch funktional eingeschränkt $\mathrm{zu}$ sein. Viele Menschen mit einer oder mehreren
Erkrankungen meistern ihren Alltag ohne jegliche Einschränkungen.

Durch altersphysiologische Prozesse nimmt mit steigendem Lebensalter die Wahrscheinlichkeit $\mathrm{zu}$, an chronischen Erkrankungen $\mathrm{zu}$ leiden (Barnett, Mercer, Norbury, Watt, Wyke, \& Guthrie 2012). Zum Beispiel erhöht die Abnahme der Knochendichte und der Muskelkraft das Risiko für Muskelskeletterkrankungen wie Osteoporose oder einen Bandscheibenvorfall. Auch erhöht die Abnahme von Arterienelastizität und Herzschlagvolumen die Wahrscheinlichkeit von Herzkreislauferkrankungen. Gleichzeitig kumulieren Risikofaktoren wie Rauchen oder Übergewicht über das Lebensalter hinweg und können zu einer erhöhten Prävalenz von Erkrankungen wie Krebs und Bluthochdruck beitragen. Es ist demnach davon auszugehen, dass im hohen Lebensalter mehr Menschen von Multimorbidität betroffen sind als in jüngeren Jahren. Aber auch im mittleren Erwachsenenalter werden bereits vermehrt chronische Erkrankungen berichtet (Barnett, Mercer et al. 2012). Diagnostizierte Erkrankungen müssen aber nicht bedeuten, dass man im Alltag gesundheitlich eingeschränkt ist. Häufig stellen die chronischen Erkrankungen zunächst aufgrund guter medikamentöser Einstellung sowie präventiver Maßnahmen (zum Beispiel Bewegung) keine Einschränkungen im Leben der Betroffenen dar. Erst später im Lebenslauf kommt es durch altersphysiologische Prozesse und durch das kumulierte Auftreten von Multimorbidität sowie von Risikofaktoren (zum Beispiel Rauchen, Feinstaubbelastung) zu funktionalen Einschränkungen (Aarts, van den Akker, Bosma, Tan, Verhey, Metsemakers, \& van Boxtel 2012).

Frauen sind häufiger von Multimorbidität betroffen als Männer (Fuchs, Busch, Lange, \& Scheidt-Nave 2012) und berichten auch mehr funktionale Einschränkungen als Männer (Menning \& Hoffmann 2009). Gründe dafür liegen zum einen in den gesundheitlichen Nachteilen eines geringeren Bildungs- und Einkommensniveaus von Frauen (vgl. Kapitel 6). Zum anderen erleben Frauen in ihrem Lebenslauf größere physiologische und hormonelle Veränderungen (Schwangerschaft, Menopause) als Männer. Frauen haben unter anderem dadurch 
ein anderes Krankheitsspektrum als Männer, sie sind zum Beispiel häufiger von Krankheiten, die die Mobilität beeinträchtigen (muskuloskelettale Erkrankungen wie Arthrose und Osteoporose), betroffen (Menning \& Hoffmann 2009; Pietschmann, Rauner, Sipos, \& KerschanSchindl 2008), die unter anderem eine Ursache für funktionale Einschränkungen sein können.

Auch unterscheidet sich die Gesundheit nach dem Bildungsniveau einer Person. Menschen mit niedriger Bildung besitzen weniger Ressourcen zur Aufrechterhaltung der Gesundheit, wie finanzielle Mittel, soziale Beziehungen oder Wissen über Gesundheit und Gesundheitsverhalten. Hinzu kommen möglicherweise auch stärkere körperliche Belastungen im Erwerbsleben. Aufgrund von geringerem gesundheitlichem Vorwissen und weniger umfassenden Krankenversicherungen (zum Beispiel weniger private Zusatzversicherungen, weniger finanzielle Möglichkeiten für Hilfsmittel) kann es auch $\mathrm{zu}$ einer schlechteren medizinischen Versorgung Niedriggebildeter kommen. Dementsprechend haben Niedriggebildete ein größeres Risiko für mehrere Krankheiten (Nagel, Peter, Braig, Hermann, Rohrmann, \& Linseisen 2008; Robert Koch-Institut 2015). Außerdem haben Personen mit geringer Bildung auch eine schlechtere Lungenfunktion als Menschen mit höherer Bildung (Hegewald \& Crapo 2007). Es ist zudem auch von stärkeren funktionalen Einschränkungen bei Niedriggebildeten auszugehen (Huisman, Kunst, Deeg, Grigoletto, Nusselder, \& Mackenbach 2005; Robert KochInstitut 2015).

Mit der steigenden Lebenserwartung stellt sich die Frage, ob die gewonnene Lebenszeit in Krankheit oder in guter Gesundheit verbracht wird. Dazu gibt es in der Forschung unterschiedliche Thesen. Morbiditätsexpansion bedeutet, dass die Anzahl an Jahren mit Erkrankungen schneller zunimmt als die Lebenserwartung insgesamt (Gruenberg 1977): Wir leben zwar länger, müssen dafür aber einen überproportionalen Zuwachs an Jahren in Krankheit in Kauf nehmen. Morbiditätskompression heißt, dass der Zuwachs an gesunden Lebensjahren im Alter größer ist als der Zuwachs an Lebenserwartung (Fries 1980). Das bedeutet, dass wir länger leben und gleichzeitig auch einen größeren Anteil unseres Lebens in Gesundheit verbringen. Beim dynamischen Äquilibrium wiederum geht man davon aus, dass zwar die Anzahl der Erkrankungen steigt, diese aber besser behandelbar sind und damit weniger Leid und weniger funktionale Einschränkungen verursachen. Es entsteht ein dynamisches Gleichgewicht zwischen dem $\mathrm{Zu}$ wachs an Gesamtlebenszeit und dem Zuwachs an Lebensjahren mit leichteren Gesundheitsbeeinträchtigungen (Manton 1982). Im vorliegenden Kapitel werden mit den DEAS-Daten in diesem Zusammenhang sowohl Aspekte der Morbidität als auch der Funktionalität ohne Berücksichtigung der Lebenserwartung analysiert. Es wird also untersucht, ob es zwischen den Kohorten Unterschiede in der Krankheitslast gibt. Zudem wird analysiert, ob sich die Veränderungen bei den selbstberichteten Krankheiten und Einschränkungen auch in einem medizinisch objektiven Maß, der Lungenfunktion, spiegeln.

Insbesondere für das Gesundheitssystem, die Pflegeversicherung und die Rentenpolitik ist es wichtig zu wissen, ob nachfolgende Kohorten eine bessere Gesundheit berichten, das heißt weniger selbstberichtete Erkrankungen und weniger funktionale Einschränkungen, als ältere Kohorten. Aufgrund des medizinischen Fortschritts, des höheren Bildungsgrades und eines gesünderen Lebensstils nachfolgender Kohorten ist mit einer Verbesserung des Gesundheitszustandes von $1996 \mathrm{zu} 2014 \mathrm{zu}$ rechnen. In der Literatur finden sich mit den Daten des Soziooekonomischen Panels (SOEP) weniger funktionale Einschränkungen bei jüngeren Kohorten von 1997 bis 2010 (Trachte, Sperlich, \& Geyer 2015). Von 2006 zu 2012 dagegen zeigte sich ebenfalls mit den Daten des SOEP ein altersdifferenziertes Bild (Steiber 2015): Während bei den 50 - bis 64-Jährigen die funktionale Gesundheit abnahm, blieb sie bei den 65 - bis 90 -Jährigen stabil. In anderen Studien in Deutschland deutet sich eine Zunahme der gesunden Lebenserwartung unabhängig vom Alter an (Kroll \& Ziese 2009). Ältere Befunde weisen zudem darauf hin, dass sich auch die Lungenfunktion von $1965 \mathrm{zu}$ 1990 in nachfolgenden Kohorten verbessert hat (Xu, Laird, Dockery, Schouten, Rijcken, \& Weiss 1995). In Statistiken aus verschiedenen europä- 
ischen Ländern, den USA und Japan, zeigt sich häufig eine erhöhte Morbidität bei gleichzeitiger Abnahme von funktionalen Einschränkungen in nachfolgenden Kohorten, was für ein dynamisches Gleichgewicht spricht (Klijs, Mackenbach, \& Kunst 2011; Doblhammer \& Kreft 2011). Es gibt aber auch Studien, die zeigen, dass die gesunde Lebenserwartung in vielen Ländern weniger schnell steigt als die generelle Lebenserwartung, was auf eine Zunahme der Morbidität hindeutet (Salomon, Wang, Freeman, Vos, Flaxman, Lopez, \& Murray 2013).

Neben dem allgemeinen Trend ist es wichtig zu wissen, ob alle Bevölkerungsgruppen gleichermaßen von diesem Wandel betroffen sind. Zum Beispiel könnte sich die Gesundheit von Erwachsenen im mittleren Alter durch weniger körperliche Belastungen in der Arbeitswelt über die Kohorten verbessern, während höhere Altersgruppen von diesem Wandel (noch) nicht profitieren. Für Männer und Frauen könnte der Wandel durch eine Angleichung der Lebensbedingungen in nachfolgenden Kohorten geprägt sein: Frauen, die ohnehin von mehr Erkrankungen und Einschränkungen betroffen sind, könn- ten durch eine steigende Erwerbsbeteiligung und die damit verbundenen Belastungen in jüngeren Kohorten eine schlechtere Gesundheit aufweisen. Von besonderer gesellschaftlicher Bedeutung sind Bildungsunterschiede: Es gilt die Frage zu klären, ob sich Bildungsunterschiede in nachfolgenden Kohorten eher verschärfen oder verringern. So finden sich beispielsweise Hinweise auf eine Morbiditätskompression bei Hochgebildeten und eine Morbiditätsexpansion bei Personen mit niedrigerer Bildung in den USA (Meara, Richards, \& Cutler 2008).

Das vorliegende Kapitel wird sich entsprechend mit den zwei folgenden Fragen auseinandersetzen:

1. Wie ist die Gesundheit (selbstberichtete Erkrankungen und funktionale Gesundheit) für verschiedene Bevölkerungsgruppen im Jahr 2014?

2. Wie hat sich die Gesundheit über den Erhebungszeitraum gewandelt? Sind alle Bevölkerungsgruppen gleichermaßen vom Wandel betroffen?

\subsection{Datengrundlage ${ }^{1}$}

Daten. Für die Beantwortung der Fragestellungen werden die Daten der 40 - bis 85 -Jährigen Teilnehmerinnen und Teilnehmer des DEAS aus den Jahren 1996, 2002, 2008 und 2014 genutzt. Die selbstberichteten Erkrankungen werden seit 1996 im schriftlichen Fragebogen erfasst, der Lungenfunktionstest wird seit 2008 als Teil des mündlichen Interviews durchgeführt und die funktionale Gesundheit wird im mündlichen Interview seit dem Jahr 2002 erfasst.

Selbstberichtete Erkrankungen. Die Anzahl der Erkrankungen wird im DEAS mit elf Krankheitsgruppen erfasst (Eigenentwicklung für den DEAS 1996; Wurm, Tesch-Römer, \& Tomasik

1 Die Daten des DEAS können für wissenschaftliche Zwecke kostenlos beim Forschungsdatenzentrum des DZA (www.fdz-dza.de) bezogen werden.
2007). Die Befragten wurden gebeten anzugeben, welche der folgenden Erkrankungen sie haben: Herzkreislauferkrankung; Durchblutungsstörung; Gelenk-, Knochen-, Bandscheiben- oder Rückenleiden; Atemwegserkrankung, Asthma oder Atemnot; Magen- oder Darmerkrankung; Krebserkrankung; Zucker/Diabetes; Gallen-, Leber- oder Nierenleiden; Blasenleiden; Augenleiden/Sehstörungen; Ohrenleiden/ Schwerhörigkeit. Zur Datenanalyse für das vorliegende Kapitel wurde die Anzahl der körperlichen Erkrankungen in einen Multimorbiditätsindex überführt. Das heißt, es wird bei jeder Fragestellung der Anteil an Personen, die keine oder eine Erkrankung haben, der Anteil an Personen, die eine mittlere Krankheitslast aufweisen - also zwei bis vier Erkrankungen haben - und der Anteil an Personen, die eine hohe 
Krankheitslast haben - also fünf und mehr Erkrankungen angeben - berichtet.

Lungenfunktion. Die Lungenfunktion wurde mittels des Mini-Wright Peak-Flow-Meters gemessen (Teilnahmequote 87,8 Prozent im Jahr 2008 und 90,8 Prozent im Jahr 2014; vgl. Kapitel 2). Dazu werden die Teilnehmerinnen und Teilnehmer gebeten so schnell und kräftig wie möglich in ein Spirometer auszuatmen. Gemessen wird dabei die Höchstgeschwindigkeit mit der eine Person ausatmen kann. Die berichteten Werte entsprechen Liter pro Minute. Die meisten Teilnehmerinnen und Teilnehmer liegen mit ihren Werten im Normbereich der Lungenfunktion. Im Jahr 2008 haben nur 3,3 Prozent, im Jahr 2014 nur 1,9 Prozent auffällig niedrige Werte gezeigt.

Funktionale Gesundheit. Die funktionale Gesundheit wird im DEAS mit zehn Fragen zu Einschränkungen in täglichen Aktivitäten erfasst (Subskala körperliche Funktionsfähigkeit der SF-36; Bullinger \& Kirchberger 1998; Ware \& Sherbourne 1992). Die zehn Tätigkeiten sind bei der Abfrage nach absteigender Schwierigkeit geordnet. Dabei handelt es sich um: anstrengende Tätigkeiten (zum Beispiel schnell laufen, schwere Gegenstände heben, anstrengenden Sport treiben); mittelschwere Tätigkeiten (zum Beispiel einen Tisch verschieben, staubsaugen, kegeln, Golf spielen); Einkaufstaschen heben oder tragen; mehrere Treppenabsätze steigen; einen Treppenabsatz steigen; sich beugen, knien, bücken; mehr als einen Kilometer zu Fuß gehen; mehrere Straßenkreuzungen weit zu Fuß gehen; eine Straßenkreuzung weit zu Fuß gehen; sich baden oder anziehen. Die Befragten schätzten ein, inwiefern sie bei den genannten Tätigkeiten ,überhaupt nicht ' (1), ,etwas ' (2) oder , stark ‘ (3) eingeschränkt sind. Die Antworten wurden für jede Person gemittelt. Bei den folgenden Ergebnissen wird jeweils der Anteil an Personen berichtet, die keine oder geringe Einschränkungen angeben (Mittelwert < 1,3) und damit eine gute funktionale Gesundheit berichten.

Gruppierungsvariablen. Altersunterschiede werden mit drei Altersgruppen (40 bis 54 Jahre, 55 bis 69 Jahre und 70 bis 85 Jahre) veranschaulicht. Weiterhin werden für Gruppenunterschiede das Geschlecht der Befragten und der Bildungsgrad in drei Stufen (niedrige, mittlere und hohe Bildung) genutzt, gemessen mit einer reduzierten ISCED-Klassifizierung. Altersunterschiede im Wandel werden, falls vorhanden, mit Sechs-Jahres-Altersgruppen beschrieben, damit es nicht $\mathrm{zu}$ Überschneidungen zwischen den Erhebungszeitpunkten und den Altersgruppen kommt ( 42 bis 47 Jahre, 48 bis 53 Jahre, 54 bis 59 Jahre, 60 bis 65 Jahre, 66 bis 71 Jahre, 72 bis 77 Jahre, 78 bis 83 Jahre). Weitere Informationen zu den Gruppierungsvariablen finden sich in Kapitel 2.

Analysen. Signifikanztestungen erfolgten über lineare, logistische und ordinale Regressionsmodelle unter Kontrolle der Stratifizierungsvariablen Altersgruppe (40 bis 54 Jahre, 55 bis 69 Jahre und 70 bis 85 Jahre), Geschlecht und Region (West-/Ostdeutschland). Für die Regressionen zur Überprüfung der Unterschiede bei der Lungenfunktion wurde zusätzlich zu den Stratifizierungsvariablen die Größe der Befragten kontrolliert, da die Körpergröße einen starken Einflussfaktor auf die Lungenfunktion darstellt (Pellegrino et al.). In den meisten Abbildungen werden gewichtete Ergebnisse dargestellt. Abbildung 8-2 stellt die aus den am Mikrozensus gewichteten logistischen Regressionen errechneten Prozentwerte dar.

\subsection{Gesundheit im Jahr 2014}

Im Jahr 2014 beträgt über alle Altersgruppen hinweg der Anteil an Personen, die keine oder eine Erkrankung berichten, 37,8 Prozent. Der Anteil an Personen mit zwei bis vier Erkrankungen beträgt 48,8 Prozent und der Anteil von Personen mit fünf oder mehr Erkrankungen 13,3 Prozent (Abbildung 8-1a). Es zeigen sich im Jahr 2014 deutliche Altersunterschiede bei der Anzahl der selbstberichteten Erkrankungen. Während bei den 40- bis 54-Jährigen noch gut die Hälfte (52,9 Prozent) null 
oder eine Erkrankung angeben, sind es bei den 70bis 85-Jährigen nur noch 17,9 Prozent. Betrachtet man nur die Personen, die keine Erkrankung berichten (ohne Abbildung), nimmt der Anteil noch einmal deutlich ab: In der jüngsten Altersgruppe berichten 22,2 Prozent, bei den 55- bis 69-Jährigen 12,8 Prozent und bei den 70- bis 85-Jährigen immerhin noch 4,8 Prozent gar keine Erkrankung. Dagegen ist der Anteil an Personen, die zwei bis vier sowie fünf und mehr Erkrankungen haben, in höheren Altersgruppen größer. Unter den 40 - bis 54-Jährigen berichten nur 5,1 Prozent fünf und mehr Erkrankungen, bei den 55- bis 69-Jährigen sind es immerhin 14,3 Prozent und unter den 70- bis 85-Jährigen berichtet ein Viertel der Personen (25,4 Prozent) fünf und mehr Erkrankungen. Aufgrund von altersphysiologischen Prozessen bestehen auch deutliche Unterschiede bei der Lungenfunktion. Die 40- bis 54-Jährigen erreichen im Durchschnitt einen Wert von 457,7 l/min, die 55- bis 69-Jährigen einen Wert von 405,9 1/min und die 70- bis 85-Jährigen haben im Schnitt einen Wert von 323,5 1/min (vgl. Tabelle A 8-3 im Anhang).
Ähnlich wie bei den Erkrankungen und der Lungenfunktion besteht auch beim Anteil an Personen mit guter funktionaler Gesundheit, wie erwartet, ein Altersunterschied (Abbildung 8-1b). Von den 40- bis 54-Jährigen berichten 83,5 Prozent eine gute funktionale Gesundheit. Dieser Anteil liegt bei den 55- bis 69-Jährigen bei 66,8 Prozent. Immerhin knapp die Hälfte der 70- bis 85 -Jährigen berichtet noch eine gute funktionale Gesundheit (48,4 Prozent).

Der Anteil an Personen mit null oder einer, zwei bis vier sowie fünf und mehr Erkrankungen unterscheidet sich nicht zwischen Männern und Frauen (Abbildung 8-1a). Der erwartete Geschlechtsunterschied zeigt sich also nicht für die selbstberichteten Erkrankungen. Die Lungenfunktion dagegen unterscheidet sich wegen biologischer Voraussetzungen zwischen Männern und Frauen (486,7 1/min versus 330,0 1/min; vgl. Tabelle A 8-3 im Anhang). Männer berichten zu einem größeren Anteil (73,9 Prozent) eine gute funktionale Gesundheit als Frauen (64,2 Prozent; Abbildung 8-1b). Es sind also wie erwartet mehr Frauen von funktionalen Einschränkungen betroffen als Männer.

Abbildung 8-1: Anzahl selbstberichteter Erkrankungen und Anteile der Personen mit guter funktionaler Gesundheit, gesamt, nach Alter, Geschlecht und Bildung, im Jahr 2014 (in Prozent)

a) Anzahl selbstberichteter Erkrankungen

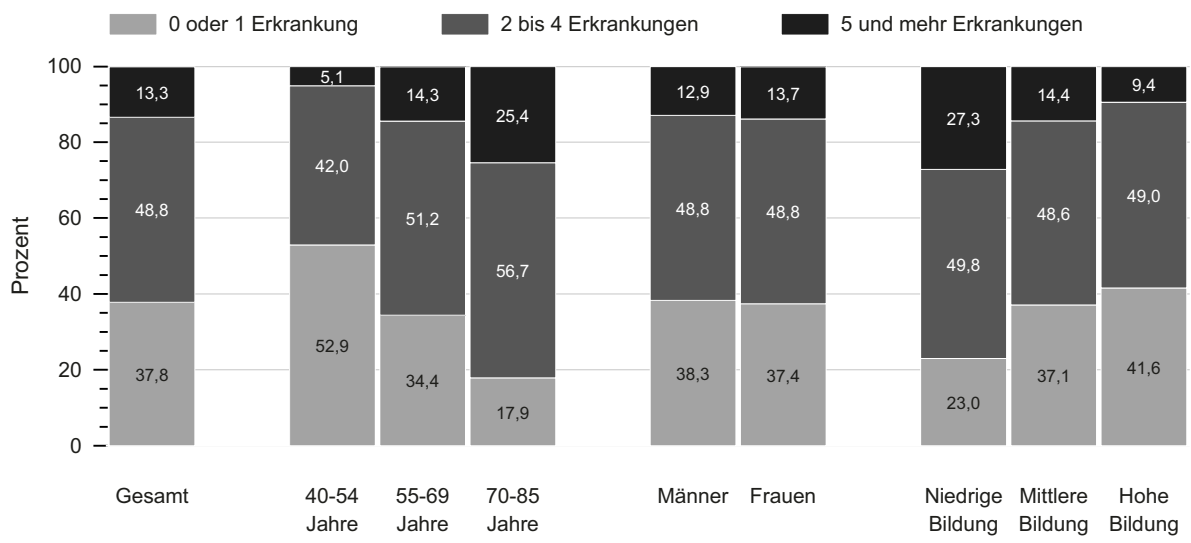




\section{b) Anteile der Personen mit guter funktionaler Gesundheit}

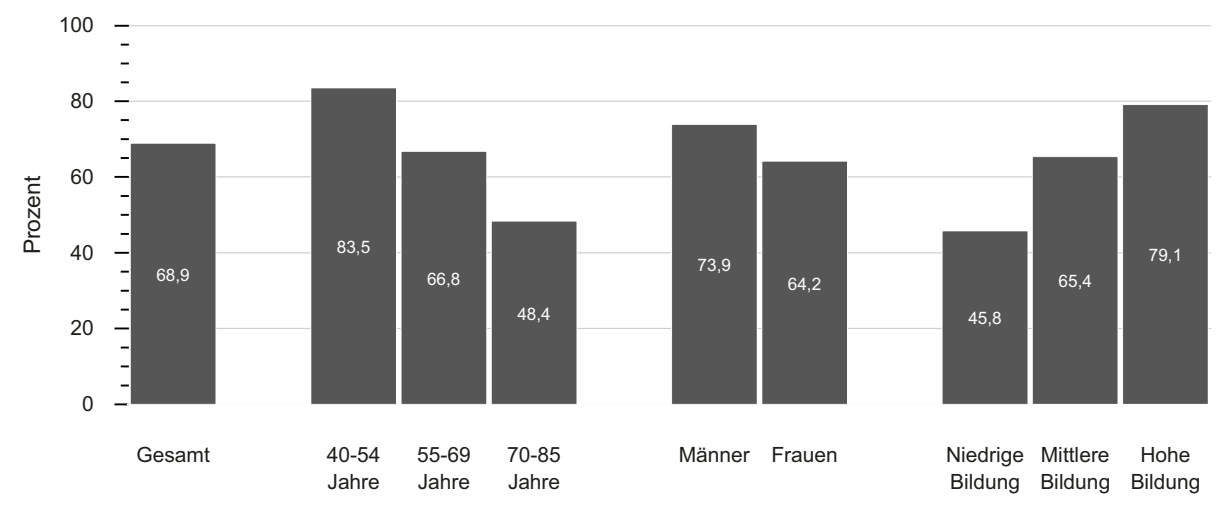

Quelle: DEAS 2014 (Selbstberichtete Erkrankungen: $n=4.219$; funktionale Gesundheit: $n=5.997$ ) gewichtet, gerundete Angaben; $(p<, 05)$.

a) Signifikante Altersgruppenunterschiede. Keine signifikanten Geschlechtsunterschiede. Signifikante Bildungsgruppenunterschiede in allen Kategorien außer im Anteil an Personen mit zwei bis vier Erkrankungen zwischen Personen mit mittlerer und hoher Bildung. b) Altersgruppen-, Geschlechts- und Bildungsgruppenunterschiede signifikant.

\section{Bei der Gesundheit bestehen im Jahr 2014 große Bildungsunterschiede.}

Auch die Bildungsunterschiede entsprechen 2014 den Erwartungen (Abbildung 8-1a und 8-1b). Der Anteil an Personen mit keiner oder einer Erkrankung ist geringer bei Niedriggebildeten (23,0 Prozent) im Vergleich zu Personen mit mittlerer oder hoher Bildung (37,1 beziehungsweise 41,6 Prozent; Abbildung 8-1a). Auch Personen mit mittlerer und hoher Bildung unterscheiden sich hier bedeutsam. Der Anteil an Personen mit zwei bis vier Erkrankungen ist bei Personen mit niedriger Bildung im Vergleich zu Personen mit mittlerer beziehungsweise hoher Bildung etwas höher, während sich Menschen mit mittlerer und hoher Bildung hier nicht signifikant unterscheiden. Der Anteil von Personen mit fünf und mehr Erkrankungen ist in höheren Bildungsgruppen geringer. Alle Bildungsgruppen unterscheiden sich dabei signifikant voneinander. Das bedeutet, dass Niedriggebildete häufiger mehrere Krankheiten berichten als Personen mit mittlerem oder hohem Bildungsniveau. Auch die Lungenfunktion von Personen mit höherer Bildung ist besser als die von Personen mit niedrigerer Bildung. Im Durchschnitt erreichen Personen mit niedriger Bildung einen Wert von $299,3 \mathrm{l} / \mathrm{min}$, Personen mit mittlerer Bildung einen Wert von 393,8 1/min und Personen mit hoher Bildung haben im Schnitt einen Wert von 448,4 1/min (ohne Abbildung). Mit höherer Bildung steigt auch der Anteil an Menschen, die eine gute funktionale Gesundheit berichten (Abbildung 8-1b). Während die Niedriggebildeten nur zu 45,8 Prozent eine gute funktionale Gesundheit berichten, sind es bei den Personen mit mittlerer Bildung 65,4 Prozent und bei den Hochgebildeten 79,1 Prozent. Das heißt, dass Niedriggebildete häufiger funktionale Einschränkungen berichten als Personen mit mittlerer oder höherer Bildung.

\section{Trotz eines hohen Anteils von Personen mit Mehrfacherkrankungen berichten viele der 40- bis 85-Jährigen im Jahr 2014 eine gute funktionale Gesundheit.}

Auch wenn gut 60 Prozent der 40 - bis 85 -Jährigen mehr als eine Erkrankung berichten, heißt dies nicht unbedingt, dass diese Personen auch funktional in ihrem Alltag eingeschränkt sind. 
Um zu überprüfen, inwiefern sich der Anteil an Personen mit guter funktionaler Gesundheit unter den Menschen mit null oder einer, zwei bis vier oder fünf und mehr Erkrankungen unterscheidet, wurde der Zusammenhang der beiden Gesundheitsdimensionen untersucht. Dieser Zusammenhang erwies sich als statistisch signifikant: Personen, die mehr Erkrankungen berichten, geben seltener eine gute funktionale Gesundheit an. Allerdings berichten unter den Personen mit zwei bis vier Erkrankungen noch gut zwei Drittel eine gute funktionale Gesundheit (68,3 Prozent) und selbst die Personen mit fünf und mehr Erkrankungen geben noch zu 39,2 Prozent eine gute funktionale Gesundheit an (Abbildung 8-2).

\section{Abbildung 8-2: Anteile der Personen mit guter funktionaler Gesundheit nach der Anzahl an} selbstberichteten Erkrankungen, im Jahr 2014 (in Prozent)

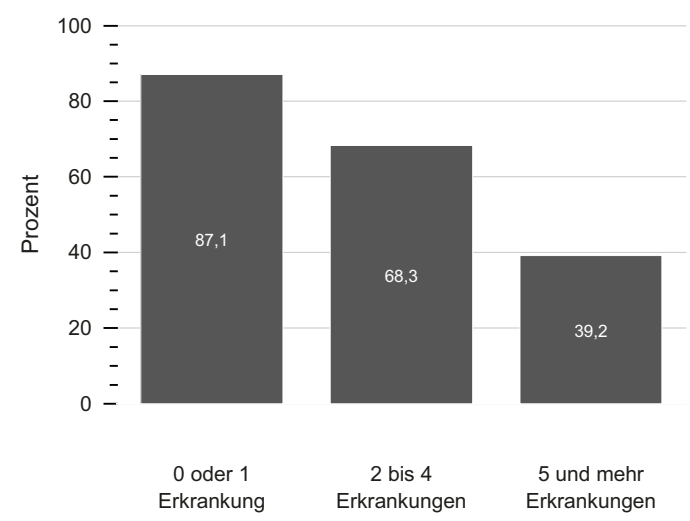

Quelle: DEAS 2014 ( $n=4.219)$ gewichtet, gerundete Angaben; $(p<, 05)$.

Signifikanter Zusammenhang zwischen selbstberichteten Erkrankungen und guter funktionaler Gesundheit. Signifikante Unterschiede in der funktionalen Gesundheit zwischen allen Kategorien der selbstberichteten Erkrankungen.

\subsection{Der Wandel der Gesundheit über die Jahre 1996, 2002, 2008 und 2014}

Bisher hatten sich in den Daten des DEAS von 1996 bis 2008 positive Ergebnisse im Wandel der Gesundheit gezeigt. Jüngere Kohorten hatten weniger Erkrankungen (1996 bis 2008) und eine bessere funktionale Gesundheit berichtet als ältere Kohorten (2002 bis 2008; Wurm, Schöllgen, \& Tesch-Römer 2010).

\section{Der Wandel hin zu weniger selbstberichteten Erkrankungen von 1996 zu 2008 setzt sich im Jahr 2014 nicht fort.}

Unterschiede zwischen den Erhebungsjahren 1996 bis 2014 in dem Anteil an Personen mit null oder einer, zwei bis vier sowie fünf und mehr Erkrankungen sind in Abbildung 8-3 dargestellt. Im Mittel hat der Anteil an Personen, die keine oder eine Erkrankung berichten, von 1996 (35,9 Prozent) zu 2014 (37,8 Prozent) zugenommen. Allerdings zeigt sich von 2002 zu 2008 ein Plateau auf hohem Niveau (circa 44 Prozent), gefolgt von einer Abnahme zum Jahr 2014. Der Anteil an Personen mit zwei bis vier Erkrankungen und der an Personen mit fünf und mehr Erkrankungen hat sich über 
die Erhebungsjahre gleichermaßen verändert. Damit setzt sich der positive Trend zu weniger selbstberichteten Erkrankungen, der sich bis 2008 gezeigt hatte, im Jahr 2014 nicht fort. Der Wandel unterscheidet sich nicht bezüglich der Altersgruppen, des Bildungsniveaus oder des Geschlechts. Das heißt, die Veränderungen über die Erhebungsjahre betreffen alle untersuchten gesellschaftlichen Gruppen gleichermaßen und die Gruppen unterscheiden sich nur in der Höhe der prozentualen Anteile in den drei Krankheitsgruppen.

Abbildung 8-3: Anzahl an selbstberichteten Erkrankungen, in den Jahren 1996, 2002, 2008 und 2014 (in Prozent)

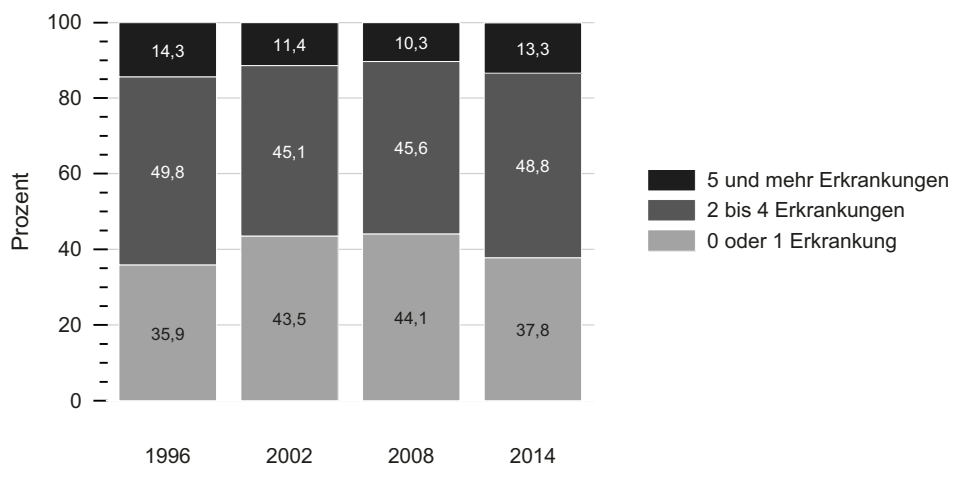

$\overline{\text { Quelle: DEAS } 1996}(n=4.003), 2002(n=2.775), 2008(n=4.352), 2014(n=4.219)$ gewichtet, gerundete Angaben; $(p<, 05)$.

Signifikante Unterschiede zwischen 1996 und allen anderen Erhebungsjahren (2002, 2008 bzw. 2014) in allen Krankheitskategorien. Stabilität von 2002 zu 2008 und signifikante Unterschiede von 2008 zu 2014 in allen Krankheitskategorien.

Die Lungenfunktion hat sich im Gegensatz zu den selbstberichteten Erkrankungen von 2008 zu 2014 leicht von 401,0 l/min auf 406,8 1/min verbessert (vgl. Tabelle A 8-3 im Anhang). Das entspricht einem prozentualen Anstieg von 1,5 Prozent. Die Veränderung unterscheidet sich nicht nach den Altersgruppen und betrifft alle Bildungsgruppen sowie beide Geschlechter gleichermaßen.

Für die funktionale Gesundheit ergibt sich im Wandel von 2002 zu 2014 für die Altersund Bildungsgruppen ein differenzierteres Bild. Der Wandel unterscheidet sich jedoch nicht für Männer und Frauen. Für fast alle Altersgruppen unter 65 Jahren ( 42 bis 47 Jahre, 54 bis 59 Jahre, 60 bis 65 Jahre) ist der Anteil an Personen, die eine gute funktionale Gesundheit berichten, von $2002 \mathrm{zu} 2008$ stabil geblieben, hat jedoch entsprechend der Entwicklung der körperlichen Erkrankungen von $2008 \mathrm{zu} 2014$ abgenommen (Abbildung 8-4).
Dabei zeigt sich zusätzlich zum Altersunterschied auch ein Unterschied zwischen den Bildungsgruppen (ohne Abbildung): Der Anteil an Personen mit guter funktionaler Gesundheit ist bei Personen mit mittlerer Bildung von $2008 \mathrm{zu}$ 2014 stärker gesunken als in den anderen beiden Bildungsgruppen, sodass sie sich im Jahr 2014 eher dem Niveau von Niedriggebildeten annähern als dem von Hochgebildeten. Somit haben sich die Bildungsunterschiede zwischen Personen mit mittlerer und hoher Bildung von 2008 zu 2014 vergrößert.

\section{Der Anteil an Personen mit guter funktionaler Gesundheit hat zwischen 2008 und 2014 zugenommen, allerdings nur bei den über 65-Jährigen.}

In den Altersgruppen ab 65 Jahren zeichnet sich hingegen konsistent über die Bildungsgruppen 
ein positives Bild ab. Es hat sich eine Zunahme des Anteils an Personen mit guter funktionaler Gesundheit von $2002 \mathrm{zu} 2014$ ( 66 bis 71 Jahre und 78 bis 83 Jahre) gezeigt, wobei der Anteil von $2008 \mathrm{zu}$
2014 stabil geblieben ist. Bei den übrigen Altersgruppen (48 bis 53 Jahre und 72 bis 77 Jahre) ist der Anteil an Personen, die eine gute funktionale Gesundheit berichten, seit 2002 stabil geblieben.

Abbildung 8-4: Anteile der Personen mit guter funktionaler Gesundheit, nach Alter, in den Jahren 2002, 2008 und 2014 (in Prozent)

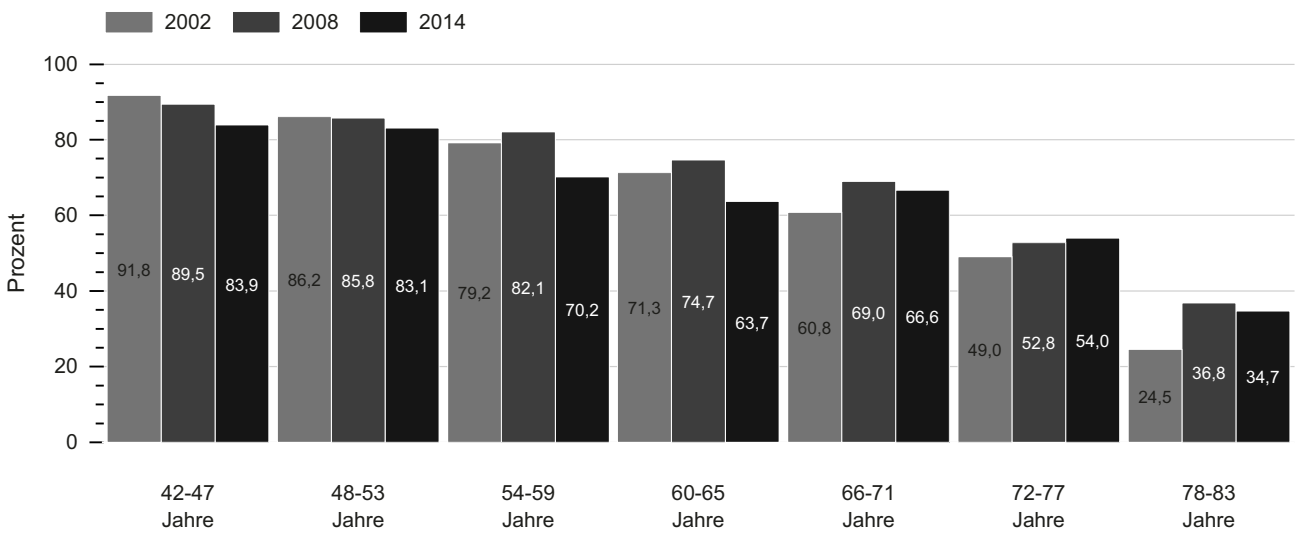

$\overline{\text { Quelle: DEAS } 2002}(\mathrm{n}=3.074), 2008(\mathrm{n}=6.202), 2014$ ( $\mathrm{n}=5.997)$ gewichtet, gerundete Angaben; $(p<, 05)$.

Stabilität von 2002 zu 2008 und signifikante Abnahme von 2008 zu 2014 für die Altersgruppen 42 bis 47 Jahre, 54 bis 59 Jahre und 60 bis 65 Jahre. Signifikante Zunahme von 2002 zu 2008 bzw. 2014 und Stabilität von 2008 zu 2014 bei den 66- bis 71-Jährigen und 78- bis 83-Jährigen. Keine Veränderung von 2002 zu 2008 und von 2008 zu 2014 bei den 48- bis 53-Jährigen und 72- bis 77-Jährigen.

\subsection{Diskussion und Implikationen}

Im Jahr 2014 zeigen sich wie erwartet deutliche Altersunterschiede bei der Gesundheit. Während das Vorhandensein mehrerer Erkrankungen bereits im mittleren Erwachsenenalter weit verbreitet ist, kommen funktionale Einschränkungen erst im hohen Alter gehäuft vor. Auch wenn mehr als eine Erkrankung vorliegt, berichten die Menschen nicht unbedingt von gesundheitlichen Einschränkungen in ihrem Alltag. Einerseits kann dies widerspiegeln, dass einige Erkrankungen gar nicht oder erst nach langen chronischen Verläufen Einschränkungen im Alltag verursachen. Andererseits spricht dieses Ergebnis für die gute medizinische Versorgung und Medikation von chronischen Erkrankungen und für ein gesundheitsbewusstes
Vorsorgeverhalten (zum Beispiel Gesundheitsverhalten, Vorsorgeuntersuchungen) von Betroffenen. Wichtig ist es, hier auch zukünftig über Präventionsmaßnahmen aufzuklären und eine gute Versorgung sicherzustellen, sodass sich funktionale Einschränkungen weiter in höhere Lebensjahre verschieben und ein längeres Leben ohne Einschränkungen möglich ist. Insbesondere eine niedrige Bildung stellt hier jedoch einen Risikofaktor dar. Im Jahr 2014 zeigen sich weiterhin deutliche Bildungsunterschiede sowohl in der Morbidität und der Lungenfunktion als auch in der funktionalen Gesundheit. Es ist Aufgabe der Politik, hier für Aufklärung in allen Bevölkerungsgruppen zu sorgen und den Zugang zu guter medizinischer Versorgung 
und Prävention auch für Personen mit niedriger Bildung und einem damit einhergehenden geringen finanziellen Spielraum sicherzustellen. Besorgniserregend ist auch, dass sich im Wandel die unter 66-Jährigen mit mittlerer Bildung in ihrer funktionalen Gesundheit eher denen mit niedriger Bildung angenähert haben als denen mit hoher Bildung. Damit ist der Vorsprung in der Gesundheit von Hochgebildeten gegenüber den anderen Bildungsgruppen zusehends größer geworden.

Betrachtet man die Gesundheit für alle Bevölkerungsgruppen im Wandel zeigt sich womöglich eine Trendwende. Der Anteil an Personen mit keiner oder einer Erkrankung, der von 1996 bis 2008 deutlich zugenommen hat, ist 2014 wieder gesunken. Für die Menschen im mittleren Alter (bis 65 Jahre) hat sich diese Wende auch in den funktionalen Einschränkungen gezeigt. Das Ergebnis entspricht nicht den Erwartungen über eine weiterhin positive Entwicklung der Gesundheit aufgrund des kontinuierlichen medizinischen Fortschritts. Nur bei den über 65-Jährigen ist entsprechend dieser Annahme der Anteil an Personen, die eine gute funktionale Gesundheit berichten, gestiegen. Auch wenn die Gesundheit nicht unter das Niveau von 1996 gesunken ist und abzuwarten ist, wie sich der Trend nach 2014 fortsetzt, kann dieses Ergebnis als Alarmsignal verstanden werden. Diese Ergebnisse stehen im Einklang mit den Befunden des SOEP über einen ähnlichen Zeitraum von 2006 zu 2012 (Steiber 2015). Auch in Frankreich kam eine Studie zu dem Schluss, dass im mittleren, nicht aber im höheren Erwachsenenalter die funktionalen Einschränkungen zugenommen haben (Cambois, Audrey, \& Robine 2012). Ein Erklärungshinweis könnte der gestiegene Anteil von Raucherinnen und Rauchern im Jahr 2014 im Vergleich zu 2008 sein (vgl. Kapitel 9). Weiterhin zeigt sich in Kapitel 12, dass der Anteil an Personen, die eine andere Person wegen gesundheitlichen Einschränkungen unterstützen, von $2008 \mathrm{zu} 2014$ zugenommen hat. Einerseits stellt dies eine gesundheitliche Belastung für die Unterstützenden dar, andererseits weist es auf einen erhöhten Unterstützungsbedarf aufgrund von gesundheitlichen Einschränkungen Älterer hin. Durch die gestiegene Erwerbsbeteiligung seit 2008 (vgl. Kapitel 3) und den gleichzeitigen Anstieg der Betreuung von Enkelkindern (vgl. Kapitel 15) sowie der Unterstützung von Personen mit gesundheitlichen Problemen (vgl. Kapitel 12) kann zudem eine Mehrfachbelastung entstehen, die insbesondere Menschen im mittleren Alter betrifft und deren Gesundheit beeinträchtigen könnte.

Die genutzte Krankheitsliste ist nicht erschöpfend und ermöglicht keine präzisen Prävalenzraten von Multimorbidität (zum Beispiel fehlen psychische und neurologische Erkrankungen). Dennoch stellt sie einen Indikator für die Krankheitslast und Beschwerdelast der Menschen im Alter von 40 bis 85 Jahren dar. Die Zunahme an selbstberichteten Erkrankungen kann aber auch ein Indiz für ein höheres Wissen über Erkrankungen, ein häufigeres Wahrnehmen von Vorsorgeuntersuchungen oder für regelmäßigere Arztbesuche sein. Die Praxisgebühr, die 2004 in Kraft trat, hielt vor allem Geringverdiener und Personen mit schlechtem Gesundheitszustand von Arztbesuchen ab (Böcken, Braun, Schnee, \& Amhof 2005). Durch die Abschaffung der Praxisgebühr im Jahr 2013 könnte es daher wieder zu vermehrten Arztbesuchen und damit Diagnosen gekommen sein, sodass der Anstieg in der Anzahl der Erkrankungen auch ein Periodeneffekt sein könnte. Auch könnte eine erhöhte Diagnostizierung von Erkrankungen durch bessere diagnostische Verfahren zu einer Erhöhung der Anzahl an selbstberichteten Krankheiten geführt haben (vgl. Moynihan, Doust, \& Henry 2012). Diese Entwicklungen können allerdings nicht die Veränderungen bei den funktionalen Einschränkungen erklären.

Trotz der höheren Anzahl an selbstberichteten Erkrankungen und einer Verschlechterung der funktionalen Gesundheit bei den unter 66-Jährigen, hat sich eine leichte Verbesserung der Lungenfunktion von 2008 zu 2014 gezeigt. Da die Lungenfunktion ein wichtiger Fitnessindikator ist, deutet dieses Ergebnis darauf hin, dass nicht alle Bereiche der Gesundheit von einer Verschlechterung betroffen sind. Es ist jedoch nicht auszuschließen, dass genau die Personen, deren Lungenfunktion schlecht und eingeschränkt ist, nicht an dem Test teilgenom- 
men haben. Zudem liegen dazu im DEAS bisher nur Daten seit 2008 vor. Aber auch in anderen Lebensbereichen, mit denen die Gesundheit in Wechselwirkung steht, sind im DEAS positive Veränderungen über die Erhebungsjahre zu verzeichnen. So hat beispielsweise das Engagement bei älteren Erwachsenen weiterhin zugenommen (vgl. Kapitel 5). Auch zeigt sich, dass nachfolgende Kohorten häufiger sportlich aktiv sind (vgl. Kapitel 9). Das heißt, dass sich die Verschlechterung der Gesundheit, insbesondere im mittleren Erwachsenenalter, von $2008 \mathrm{zu}$ 2014 nicht unbedingt in der gesellschaftlichen Teilhabe der Menschen in der zweiten Lebenshälfte widerspiegelt. Wissenschaftliche Befunde weisen allerdings daraufhin, dass insbesondere Niedriggebildete in ihren Aktivitäten im Ruhestand durch Multimorbidität eingeschränkt werden (Wetzel \& Huxhold 2016). Auch kann die erhöhte Multimorbidität langfristig zu mehr funktionalen Einschränkungen im Alltag und damit in der Aktivität der Menschen führen (Aarts, van den Akker et al. 2012). Daher gilt es weiterhin zu beobachten, wie sich die Gesundheit entwickelt und ob das hohe Aktivitätsni- veau auch zukünftig in allen gesellschaftlichen Gruppen aufrechterhalten werden kann.

Die Veränderung der Gesundheit über die Kohorten ist ein wichtiger Indikator für die Chancen und Herausforderungen einer alternden Gesellschaft. Auch mit einer Zunahme der Krankheitslast im Jahr 2014 ist die gesundheitliche Situation der heutigen Älteren besser als die Situation derer im Jahr 1996. Die gesundheitlichen Voraussetzungen für eine längere Lebensarbeitszeit in jüngeren Geburtsjahrgängen sind damit gegeben. Gleichzeitig sollte durch geeignete Präventionsmaßnahmen einer weiteren Verschlechterung der Gesundheit entgegengewirkt werden. Speziell den funktionalen Einschränkungen im Alltag kann durch bessere Gesundheitsverhaltensweisen vorgebeugt werden (vgl. auch Kapitel 9). Wenn die Gesellschaft langfristig die Gesundheit und Fitness Älterer erhalten möchte, sollte die Politik Prävention und eine gute medizinische Versorgung für die gesamte Bevölkerung gewährleisten. Dabei gilt es insbesondere sozial Benachteiligte stärker zu integrieren.

\section{Literatur}

Aarts, S., Akker, M. van den, Bosma, H., Tan, F., Verhey, F., Metsemakers, J., \& Boxtel, M. van (2012). The effect of multimorbidity on health related functioning: Temporary or persistent? Results from a longitudinal cohort study. Journal of Psychosomatic Research, 73(3), 211-217. doi: 10.1016/j.jpsychores.2012.05.014.

Barnett, K., Mercer, S. W., Norbury, M., Watt, G., Wyke, S., \& Guthrie, B. (2012). Epidemiology of multimorbidity and implications for health care, research, and medical education: a cross-sectional study. The Lancet, 380(9836), 37-43. doi: 10.1016/SO1406736(12)60482-6.

Böcken, J., Braun, B., Schnee, M., \& Amhof, R. (2005). Gesundheitsmonitor 2005: Die ambulante Versorgung aus Sicht von Bevölkerung und Ärzteschaft. Gütersloh: BertelsmannStiftung.

Bullinger, M., \& Kirchberger, I. (1998). Der SF-36 Fragebogen zum Gesundheitszustand. Handbuch für die deutschsprachige Fragebogenversion. Göttingen: Hogrefe Verlag.
Cambois, E., Audrey, B., \& Robine, J.-M. (2012). Aging and health in France: An unexpected expansion of disability in mid-adulthood over recent years. European Journal of Public Health, 23(4), 575-581. doi: 10.1093/eurpub/cks136.

Doblhammer, G., \& Kreft, D. (2011). Länger leben, länger leiden? Trends in der Lebenserwartung und Gesundheit. Bundesgesundheitsblatt, 54, 907-914. doi: 10.1007/s00103-011-1315-0.

Fries, J. F. (1980). Aging, natural death, and the compression of morbidity. New England Journal of Medicine, 303(3), 130-135. doi: 10.1056/ NEJM198007173030304.

Fuchs, J., Busch, M., Lange, C., \& Scheidt-Nave, C. (2012). Prevalence and patterns of morbidity among adults in Germany. Bundesgesundheitsblatt - Gesundheitsforschung - Gesundheitsschutz, 55(4), 576-586. doi: 10.1007/s00103-012-1464-9. 
Gruenberg, E. M. (1977). The failures of success. The Milbank Memorial Fund Quarterly. Health and Society, 55(1), 3-24. doi: 10.2307/3349592.

Hegewald, M. J., \& Crapo, R. O. (2007). Socioeconomic status and lung function. Chest, 132(5), 1608-1614. doi: 10.1378/chest.07-1405.

Huisman, M., Kunst, A., Deeg, D., Grigoletto, F., Nusselder, W., \& Mackenbach, J. (2005). Educational inequalities in the prevalence and incidence of disability in Italy and the Netherlands were observed. Journal of Clinical Epidemiology, 58(10), 1058.e-1058.e1010. doi: 10.1016/j.jclinepi.2004.12.011.

Katz, J. N., Chang, L. C., Sangha, O., Fossel, A. H., \& Bates, D. W. (1996). Can comorbidity be measured by questionnaire rather than medical record review? Medical Care, 34(1), 73-84. doi: 10.2307/3766388.

Klijs, B., Mackenbach, J. P., \& Kunst, A. E. (2011). Future disability projections could be improved by connecting to the theory of a dynamic equilibrium. Journal of Clinical Epidemiology, 64(4), 436-443. doi: 10.1016/j. jclinepi.2010.04.018.

Kroll, L. E., \& Ziese, T. (2009). Kompression oder Expansion der Morbidität? In: K. Böhm, C. Tesch-Römer \& T. Ziese (Hrsg.) Gesundheit und Krankheit im Alter. Berlin: Robert Koch-Institut.

Manton, K. G. (1982). Changing concepts of morbidity and mortality in the elderly population. The Milbank Memorial Fund Quarterly. Health and Society, 60(2), 183-244. doi: 10.2307/3349767.

Meara, E. R., Richards, S., \& Cutler, D. M. (2008). The gap gets bigger: Changes in mortality and life expectancy, by education, 1981-2000. Health Affairs, 27(2), 350360. doi: $10.1377 /$ hlthaff.27.2.350.

Menning, S., \& Hoffmann, E. (2009). Funktionale Gesundheit und Pflegebedürftigkeit. In: K. Böhm, C. Tesch-Römer \& T. Ziese (Hrsg.) Gesundheit und Krankheit im Alter (S. 62-78). Berlin: Robert Koch-Institut.

Moynihan, R., Doust, J., \& Henry, D. (2012). Preventing overdiagnosis: How to stop harming the healthy. British Medical Journal, 344, 1-6. doi: 10.1136/bmj. e3502.

Nagel, G., Peter, R., Braig, S., Hermann, S., Rohrmann, S., \& Linseisen, J. (2008). The impact of education on risk factors and the occurrence of multimorbidity in the EPIC-Heidelberg cohort. BioMed Central Public Health, 8(1), 384-394. doi: 10.1186/1471-2458-8-384.

Pellegrino, R., Viegi, G., Brusasco, V., Crapo, R. O., Burgos, F., Casaburi, R., Coates, A., Grinten, C. P. M. van der, Gustafsson, P., Hankinson, J., Jensen, R., Johnson, D. C., Maclntyre, N., McKay, R., Miller, M. R.,
Navajas, D., Pedersen, O. F., \& Wanger, J. (2005). Interpretative strategies for lung function tests. European Respiratory Journal, 26, 948-968. doi: 10.1183/09031936.05.00035205.

Pietschmann, P., Rauner, M., Sipos, W., \& KerschanSchindl, K. (2008). Osteoporosis: An age-related and gender-specific disease - a mini-review. Gerontology, 55(1), 3-12. doi: 10.1159/000166209.

Robert Koch-Institut (2015). Gesundheit in Deutschland 2015. Berlin: Robert Koch-Institut.

Salomon, J. A., Wang, H., Freeman, M. K., Vos, T., Flaxman, A. D., Lopez, A. D., \& Murray, C. J. (2013). Healthy life expectancy for 187 countries, 1990-2010: A systematic analysis for the Global Burden Disease Study 2010. Lancet, 380(9859), 2144-2162. doi: 10.1016/ s0140-6736(12)61690-0.

Steiber, N. (2015). Population aging at cross-roads: Diverging secular trends in average cognitive functioning and physical health in the older population of Germany. PlosOne, 10(8), 1-19. doi: 10.1371/journal. pone. 0136583 .

Trachte, F., Sperlich, S., \& Geyer, S. (2015). Kompression oder Expansion der Morbidität: Entwicklung der Gesundheit in der älteren Bevölkerung. Zeitschrift für Gerontologie und Geriatrie, 48, 255-262. doi: 10.1007/ s00391-014-0644-7.

Ware, J. E., Jr., \& Sherbourne, C. D. (1992). The MOS 36-item short-form health survey (SF-36): Conceptual framework and item selection. Medical Care, 30(6), 473-483. doi: 10.2307/3765916.

Wetzel, M., \& Huxhold, O. (2016). Are leisure activities and health interconnected after retirement transition? Educational differences. Advances in Life Course Research, Online first (April). doi: 10.1016/j. alcr.2016.03.007.

WHO (1946). Constitution. Genf: WHO.

Wurm, S., Schöllgen, I., \& Tesch-Römer, C. (2010). Gesundheit. In: A. Motel-Klingebiel, S. Wurm \& C. Tesch-Römer (Hrsg.) Altern im Wandel. Befunde des Deutschen Alterssurveys (DEAS) (S. 90-117). Stuttgart: Kohlhammer.

Wurm, S., Tesch-Römer, C., \& Tomasik, M. J. (2007). Longitudinal findings on aging-related cognitions, control beliefs, and health in later life. The Journals of Gerontology Series B: Psychological Sciences \& Social Sciences, 62B(3), P156-P164. doi: 10.1093/geronb/62.3.p156.

Xu, X., Laird, N., Dockery, D. W., Schouten, J. P., Rijcken, B., \& Weiss, S. T. (1995). Age, period, and cohort effects on pulmonary function in a 24-year longitudinal study. American Journal of Epidemiology, 141(6), 554-566. 
Open Access Dieses Kapitel wird unter der Creative Commons Namensnennung 2.5 International Lizenz (http://creativecommons.org/licenses/by/2.5/deed.de) veröffentlicht, welche die Nutzung, Vervielfältigung, Bearbeitung, Verbreitung und Wiedergabe in jeglichem Medium und Format erlaubt, sofern Sie den/die ursprünglichen Autor(en) und die Quelle ordnungsgemäß nennen, einen Link zur Creative Commons Lizenz beifügen und angeben, ob Änderungen vorgenommen wurden.

Die in diesem Kapitel enthaltenen Bilder und sonstiges Drittmaterial unterliegen ebenfalls der genannten Creative Commons Lizenz, sofern sich aus der Abbildungslegende nichts anderes ergibt. Sofern das betreffende Material nicht unter der genannten Creative Commons Lizenz steht und die betreffende Handlung nicht nach gesetzlichen Vorschriften erlaubt ist, ist für die oben aufgeführten Weiterverwendungen des Materials die Einwilligung des jeweiligen Rechteinhabers einzuholen. 